\title{
Inhibiting Angiotensin-Converting Enzyme Promotes Renal Repair by Limiting Progenitor Cell Proliferation and Restoring the Glomerular Architecture
}

\author{
Ariela Benigni, ${ }^{*}$ Marina Morigi, ${ }^{*}$ Paola Rizzo, ${ }^{*}$ \\ Elena Gagliardini, ${ }^{*}$ Cinzia Rota, ${ }^{*}$ Mauro Abbate, ${ }^{*}$ \\ Serena Ghezzi, ${ }^{*}$ Andrea Remuzzi, ${ }^{\dagger}$ and \\ Giuseppe Remuzzi* \\ From the Mario Negri Institute for Pharmacological Research,* \\ Centro Anna Maria Astori, Bergamo; the Industrial Engineering \\ Department ${ }^{\dagger}$ University of Bergamo, Bergamo; and the Unit of \\ Nephrology and Dialysis, ${ }^{\ddagger}$ Azienda Ospedaliera, Ospedali Riuniti \\ di Bergamo, Bergamo, Italy
}

We previously reported that angiotensin-converting enzyme inhibitor (ACEi) renoprotection in Munich Wistar Frömter (MWF) rats, which develop progressive glomerular injury, was associated with podocyte repopulation and preservation of glomerular architecture. Here, we studied the time course of the lesions, their cellular components, and the effect of ACEi. Early glomerular lesions were synechiae, followed by extracapillary crescents and glomerulosclerosis. The majority of cells forming crescents were claudin $1^{+}$parietal epithelial cells and, to a lesser extent, $\mathrm{WT}^{+}{ }^{+}$podocytes, both in active proliferation. In crescents, cells expressing the metanephric mesenchyme marker NCAM were also found. Three distinct populations of parietal epithelial cells were identified in the rat Bowman's capsule: $\mathrm{NCAM}^{+} \mathrm{WT}^{-}$cells, also expressing progenitor cell marker CD24, and $\mathrm{NCAM}^{+} \mathrm{WT1}^{+}$and $\mathrm{NCAM}^{-} \mathrm{WT}^{+}$cells, the latter population representing parietal podocytes. After exposure to inductive medium, cultured parietal epithelial cells that were obtained by capsulated glomeruli generated podocytes, documenting their progenitor nature. Mitotic activity of cultured renal progenitors was induced by angiotensin II through the down-regulation of cell cycle inhibitor C/EBP $\delta$ expression. Treatment with ACEi reduced number and extension of crescents and glomerulosclerosis in MWF rats. Renoprotection was accomplished through the limitation of $\mathrm{NCAM}^{+}$progenitor proliferation via the modulation of C/EBP $\delta$. Thus, chaotic migration and proliferation of the Bowman's capsule progenitor cells pave the way to crescent formation and subsequent sclerosis. ACEi, by moderating progenitor cell activation, restores glomerular architecture and prevents renal disease progression. (Am J Pathol 2011, 179:628-638; DOI: 10.1016/j.ajpath.2011.04.003)

Chronic kidney disease is a worldwide threat to public health. Estimates report that diseases of the kidney account for 830,000 global deaths every year, with a sharp rise of renal replacement therapy now exceeding 2 million patients for an aggregate cost of more than US\$1 trillion. ${ }^{1}$ The burden of chronic kidney disease is not limited to demand of renal replacement therapies, but chronic kidney disease is also a major determinant of cardiovascular diseases, with direct impact on the health of the overall population. ${ }^{2}$ Since there are no specific treatments for most chronic nephropathies so far, efforts aimed at preventing renal disease progression are mandatory. Studies have documented that progressive renal function deterioration is the result of compensatory glomerular hemodynamic changes in response to nephron loss. A key player is angiotensin ${ }^{3}$ to the extent that angiotensin-converting enzyme inhibitors (ACEi) or angiotensin II receptor antagonists slow the development of proteinuria and limit renal damage in animals. ${ }^{4,5}$ Robust clinical evidence of remission and regression of renal disease in humans ${ }^{6}$ was clarified by subsequent animal studies. By three-dimensional reconstruction of glomerular capillary tufts based on kidney serial section analysis,

Supported by a grant from Fondazione Cariplo (Milano, Italy; grant 20075549). The research has received funding from the European Community under the European Community's Seventh Framework Programe (FP7/ 2007-2013), grant number 223007, STAR-TREK project. C.R. is a recipient of a fellowship from Fondazione Aiuti per la Ricerca sulle Malattie Rare (ARMR), Bergamo, Italy.

Accepted for publication April 22, 2011.

A.B., M.M., and P.R. contributed equally to this work.

Address reprint requests to Paola Rizzo, Biol.Sci.D., Mario Negri Institute for Pharmacological Research, Centro Anna Maria Astori, Science \& Technology Park Kilometro Rosso, Via Stezzano, 87 - 24126 Bergamo, Italy. E-mail: paola.rizzo@marionegri.it. 
we found that after 10 weeks of ACEi treatment in Munich Wistar Frömter (MWF) rats, studied at 60 weeks, more that $30 \%$ of glomeruli were completely free of sclerosis, whereas all glomeruli of 50-week-old untreated MWF rats had some degree of scarring. ${ }^{7}$ This approach did not identify glomerular cellular components. ${ }^{8}$ Regression of glomerulosclerosis and neoformation of glomerular tissue has been linked to progenitor/stem cells of renal or extrarenal origin. ${ }^{9}$ As a follow-up of previous studies, ${ }^{7}$ we recently showed that, in MWF rats, ACEi halted the spontaneous podocyte loss ${ }^{10}$ and restored podocyte number. Consistently, others documented recruitment of podocytes from glomerular parietal epithelium toward the capillary tuft. ${ }^{11}$ A population of progenitor cells localized within the Bowman's capsule has been recently found in human adult kidney. ${ }^{12}$ These cells can regenerate podocytes. ${ }^{13}$ Renal progenitors and transitional cells-progenitor cells that additionally expressed podocyte markers-were also detected within hyperplastic lesions of human glomerulopathies. ${ }^{14}$ Mechanisms and cellular determinants of progressive nephropathies in the context of recent findings of glomerular epithelial cell activation had never been addressed in systematic fashion. Inhibiting ACE can be a selective way to potentiate the regeneration of the glomerulus, shifting the process toward kidney healing. To this end, the spontaneous glomerulopathy of MWF rats represents the most appropriate model in which to study the cellular basis for glomerular restructuring and repair. Here, we first sought to establish whether a population of progenitor cells actually exists in the rat glomerulus. We then evaluated whether renal injury in MWF could be the consequence of aberrant progenitor cell proliferation, and to what extent renoprotection by ACEi occurred via an effect of moderating progenitor cell migration and proliferation to restore the Bowman's capsule architecture.

\section{Materials and Methods}

\section{Study Design}

Sixty-four male MWF rats from our colony were divided into different groups as follows: group $1(n=50)$ received saline and were sacrificed at different time points, 10, 25, 40,50 , and 60 weeks of age $(n=10$ rats for each time point); group $2(n=10)$ received lisinopril $(80 \mathrm{mg} / \mathrm{L}$ in drinking water) from 50 to 60 weeks of age; group 3 ( $n=$ 4) received lisinopril $(80 \mathrm{mg} / \mathrm{L}$ in drinking water) from 50 to 52 weeks of age. Ten- to 60-week-old Wistar rats (Charles River S.p.A., Calco, Italy) were used as controls $(n=20)$. All rats were maintained in a room with constant temperature and light, and they had free access to water and food. Animal care and treatment were conducted in conformity with the institutional guidelines that are in compliance with national (Decreto Legislativo n116, Gazzetta Ufficiale suppl. 40, 18/2/1992, Circolare N.8, Gazzetta Ufficiale 14/7/1994) and international laws and policies (EEC Council Directive 86/609, OJL 358-1, 1987; Guide for the Care and Use of Laboratory Animals. $\mathrm{NIH}$ publication n. 85-23. Revised 1996). All animal stud- ies were approved by the Institutional Animal Care and Use Committees of "Mario Negri" Institute for Pharmacological Research. Blood pressure was evaluated by tail plethysmography in awake animals, and urinary protein excretion was monitored during the study by Coomassie Blue $\mathrm{G}$ dye-binding assay ${ }^{15}$ in 24-hour urine collections. Serum creatinine was measured during the observation period by the Jaffe colorimetric test. ${ }^{16}$ At sacrifice, kidneys were perfused with PBS under anesthesia, collected, fixed, and then processed for histology and immunohistochemistry as described. To study cell proliferation, rats given vehicle or lisinopril from 50 to 60 weeks of age ( $n=4$ for each group) were injected intraperitoneally with an S-phase labeled 5-bromo-2'-deoxyuridine (BrdU) (Sigma Aldrich, St Louis, MO, 50 mg/kg dissolved in saline) for 5 days before sacrifice.

\section{Renal Histology}

The removed kidneys were fixed in Duboscq-Brazil and embedded in paraffin. Three-micron sections were stained with periodic acid-Schiff reagent and observed by light microscopy (BH2-RFCA; Olympus, Melville, NY). At least 50 glomeruli were examined for each animal, and the extent of synechiae or crescents was expressed by giving a score from 0 to 4 related to the percentage of glomerular tuft occupied by the lesions (0: no lesions, 1: lesions affecting $<25 \%$ of the glomerulus, 2: lesions affecting $25 \%$ to $50 \%$ of the glomerulus, 3 : lesions affecting $50 \%$ to $75 \%$ of the glomerulus, and 4 : lesions affecting $75 \%$ to $100 \%$ of the glomerulus). The indices of synechiae and crescents were calculated by using the following formula:

$$
\text { Index }=\frac{\left(1 \times n_{1}\right)+\left(2 \times n_{2}\right)+\left(3 \times n_{3}\right)+\left(4 \times n_{4}\right)}{\left(n_{0}+n_{1}+n_{2}+n_{3}+n_{4}\right)}
$$

where $n_{x}$ is the number of glomeruli with each percentage of lesion extension.

Renal samples were analyzed by the same observer, who was unaware of the nature of the different experimental groups.

\section{Immunofluorescence Experiments}

Claudin1, Wilms' tumor-1 (WT1), neural cell adhesion molecule (NCAM), Thy1.1, ED1, and BrdU expression was detected by immunofluorescence analysis. Four percent paraformaldehyde-fixed cryosections were air dried, washed with PBS $1 \times$ and incubated with $1 \%$ bovine serum albumin (BSA) to block nonspecific sites. The following primary antibodies were used: rabbit anti-claudin1 (undiluted; Thermo Scientific, Rockford, IL), rabbit or mouse anti-WT1 (1:50; Santa Cruz Biotechnology, Santa Cruz, CA), mouse anti-NCAM (1:4; Developmental Studies Hybridoma Bank, University of lowa, lowa City, IA), mouse anti-CD24 (1:25; BD Pharmingen, Franklin Lakes, NJ), mouse anti-Thy1.1 (1:100; AbD Serotec, Kidlington, Oxford, UK), mouse anti-ED1 (1:100; Chemicon, Temecula, CA), fluorescein isothiocyanate (FITC)-conju- 
gated mouse anti-BrdU (1:4; Roche Diagnostic GmbH, Mannheim, Germany), mouse anti-BrdU (Becton Dickinson Immunocytometry Systems, San Jose, CA), followed by the specific FITC- or Cy3-conjugated secondary antibodies. For mouse anti-NCAM and mouse anti-CD24 double staining, specific anti-lgG1 and anti-IgM secondary antibodies were used. Nuclei were stained with DAPI, and the renal structure with FITC wheat germ agglutinin. Negative controls were obtained by omitting primary antibodies on adjacent sections. For the detection of claudin1, WT1, NCAM, CD24, and BrdU, antigen retrieval was performed in citrate buffer $10 \mathrm{mmol} / \mathrm{L}(\mathrm{pH} \mathrm{6.0)}$ ) at boiling temperature for 20 minutes, followed by incubation with citrate buffer (20 minutes) at room temperature to enhance the reactivity of antibodies to antigens. Fluorescence was examined by an inverted confocal laser scanning microscope (LS 510 Meta; Zeiss, Jena, Germany). All glomeruli were acquired and subjected to quantitative analysis. The percentage of positive cells was evaluated based on the total number of $\mathrm{DAPI}^{+}$cells within crescents or in Bowman's capsule.

\section{Immunoperoxidase Experiments}

Type III collagen and CCAAT/enhancer binding protein (C/EBP $\delta$ ) expression was detected by immunoperoxidase analysis. Duboscq-Brazil-fixed, paraffin-embedded kidney sections ( $3 \mu \mathrm{m}$ ) were deparaffinized, rehydrated, and then incubated for 30 minutes with $0.3 \% \mathrm{H}_{2} \mathrm{O}_{2}$ in methanol to quench endogenous peroxidase. Antigen retrieval was performed using a microwave $[2 \times 5$ minutes in citrate buffer $10 \mathrm{mmol} / \mathrm{L}(\mathrm{pH} \mathrm{6.0)}$ ) at an operating frequency of $2450 \mathrm{MHz}$ and a $600 \mathrm{~W}$ power output] and citrate buffer incubation (15 minutes at room temperature) to increase the reactivity of antibodies to antigens. After blocking with $1 \% \mathrm{BSA}$, sections were incubated with rabbit anti-type III collagen (1:100; Chemicon) or rabbit anti- C/EBP $\delta$ antibody (1:100; Santa Cruz Biotechnology), followed by specific biotinylated secondary antibodies and diaminobenzidine (Merck, Darmstadt, Germany) substrate solution. Slides were finally counterstained with hematoxylin, dehydrated in graded alcohols, mounted with coverslips, and observed by light microscopy (BH2RFCA; Olympus). Negative controls were obtained by omitting the primary antibody on adjacent sections. $\mathrm{C} / \mathrm{EBP} \delta$ signal was graded on a scale of 0 to 3 (0: no C/EBP $\delta$-positive cells, 1: $<25 \%$ positive cells, $2: 25 \%$ to $50 \%$ positive cells, 3: $>50 \%$ positive cells in the Bowman's capsule).

\section{Immunoelectron Microscopy}

For immunogold staining, the kidney was fixed with 3.5\% paraformaldehyde plus $0.01 \%$ glutaraldehyde overnight at $4^{\circ} \mathrm{C}$. Fragments of fixed renal tissue were embedded in Lowicryl resin (Electron Microscopy Sciences, Hatfield, PA), sectioned with an ultramicrotome (Leica Microsystems, Wetzlar, Germany), and then transferred to nickel grids coated with Formvar (Electron Microscopy Sciences, Hatfield, PA). After blocking with 1\% BSA for 15 minutes, sections were incubated overnight with goat anti-nephrin (1:400; Santa Cruz Biotechnology) followed by $12-n m$ gold-conjugated donkey anti-goat secondary antibody for 1 hour at room temperature. The grids were washed with PBS, stained for 5 minutes with $2 \%$ aqueous uranyl acetate, and examined with a Morgagni 268D electron microscopy (Philips, Brno, Czech Republic).

\section{In Vitro Experiments}

Kidneys of adult Wistar rats were quickly removed, and the homogenate of the cortex was gently pressed through a 105- $\mu \mathrm{m}$ (140 mesh) sieve and then over a $75-\mu \mathrm{m}$ (140 mesh) sieve that retains glomeruli. Glomeruli were centrifuged, resuspended in medium plus endothelial growth medium-microvascular (EGM-MV; Cambrex Bio Science, East Rutherford, NJ) and $20 \%$ fetal bovine serum (FBS, Hyclone, Logan UT), and plated on fibronectin-coated dishes ( $10 \mu \mathrm{g} / \mathrm{mL}$; Sigma-Aldrich) at a density of 200 glomeruli/100-mm plate. After 5 to 7 days of culture, adherent capsulated glomeruli showed cellular outgrowth, which was picked and cultured in EGM-MV 20\% fetal bovine serum on glass coverslips coated with fibronectin.

Claudin1, NCAM, and CD24 were detected by immunofluorescence analysis on cells from initial outgrowth of capsulated glomeruli. Cells were fixed in $2 \%$ paraformaldehyde plus $4 \%$ sucrose, permeabilized with $0.3 \%$ Triton-X100 (Sigma-Aldrich), and then blocked with $2 \%$ BSA, $0.2 \%$ gelatin bovine, and $2 \%$ FBS in PBS and then incubated 1 hour with rabbit anti-claudin1 (undiluted) or mouse anti-NCAM (1:2), followed by the specific Cy3- or FITC-conjugated secondary antibody. For CD24 expression, cells were incubated with mouse anti-CD24 antibody overnight at $4^{\circ} \mathrm{C}(1: 25)$ and then with specific FITCconjugated secondary antibody.

For differentiation and proliferation experiments, cells from glomerular outgrowth were expanded and claudin $1^{+}$cells obtained by immunomagnetic separation using goat anti-rabbit IgG MicroBeads (Miltenyi Biotec, Bergisch Gladbach, Germany) according to the manufacturer's protocol. Claudin $1^{+}$cell fraction (second passage) was used for all experiments. For podocyte differentiation, claudin $1^{+}$parietal epithelial cells (PECs) were treated for 7 days with DMEM/F12 supplemented with vitamin $D_{3} 100 \mathrm{nmol} / \mathrm{L}$ and retinoic acid $100 \mu \mathrm{mol} / \mathrm{L}$ (VRAD medium). ${ }^{13,17}$ Cells were then fixed, permeabilized, and incubated with mouse anti-synaptopodin (SYN) antibody (undiluted; ProGen, Heidelberg, Germany) and goat anti-mouse FITC. The proliferation of immunoisolated claudin $1^{+}$PECs was evaluated by studying the phosphorylation at Ser10 of histone $\mathrm{H} 3^{18}$ in cells maintained overnight in medium plus 1\% FBS and then exposed to medium or to angiotensin II $\left(10^{-7} \mathrm{~mol} / \mathrm{L}\right.$; SigmaAldrich) for 24 hours. After fixation, cells were incubated with phospho-histone $\mathrm{H} 3(\mathrm{H} 3 \mathrm{p})$ antibody followed by FITC secondary antibody. Nuclei were counterstained with DAPI. The quantification of cells positive for synaptopodin or $\mathrm{H} 3 p$ was performed counting fluorescent cells per total DAPI ${ }^{+}$cells in each high-power field (HPF) (five to 10 fields/slide; $n=3$ experiments). Data were ex- 
pressed as percentage of $\mathrm{SYN}^{+} \mathrm{H}_{3} \mathrm{p}^{+}$cells/total $\mathrm{DAPI}^{+}$ cells/HPF.

The expression of C/EBP $\delta$ was evaluated in PECs incubated in DMEM (Gibco BRL, Grand Island, NY) plus $2 \%$ BSA with or without angiotensin II $\left(10^{-7} \mathrm{~mol} / \mathrm{L}\right)$. After 6 hours, the cells were fixed and processed as above. PECs were incubated 3 hours with rabbit anti-C/EBP $\delta$ antibody (1:25) followed by FITC-conjugated secondary antibody. Nuclei were stained with DAPI, and images were captured using an inverted confocal laser scanning microscope.

\section{Statistical Analysis}

Results were expressed as mean \pm SE. Correlation analysis between index of crescents and proteinuria was performed by evaluating Pearson's $r$ coefficient, using a Microsoft Excel (Redmonds, WA) spreadsheet. Statistical analysis of proteinuria levels, synechiae, and crescents was performed using analysis of variance with the Bonferroni post hoc analysis for multiple comparisons. The nonparametric Kruskal-Wallis and Mann-Whitney tests were applied, as appropriate. Statistical significance was defined as $P<0.05$.

\section{Results}

\section{A Population of Renal Progenitor Cells Exists within the Bowman's Capsule of Normal Adult Rat Kidney}

The phenotype of PECs in the Bowman's capsule of control Wistar rats was assessed by simultaneously staining claudin1, a marker of glomerular PECs that localizes to intercellular tight junctions, ${ }^{19}$ and the podocyte marker WT1. All of the cells lining the Bowman's capsule expressed claudin 1 , and a small fraction co-expressed WT1 $(21.9 \% \pm 0.5 \%)$ (Figure $1 \mathrm{~A})$. Immunoelectron microscopy analysis allowed us to identify some PECs exhibiting foot processes in which marked expression of nephrin was found (Figure 1B). Bowman's capsule in humans is lined by a subset of parietal epithelial cells with stem cell characteristics, ${ }^{12}$ which in physiological conditions gradually lose their stemness and regenerate podocytes. ${ }^{11}$ In rats, no stem cell population in the Bowman's capsule has been described so far. In search of markers of stemness in rat, we focused on NCAM, a protein expressed in metanephric mesenchyme and in the glomerular capsule in mature kidney. ${ }^{20,21}$ In controls, NCAM was expressed by the majority of claudin $1^{+}$cells in the Bowman's capsule $(88.0 \% \pm 6.3 \%$, Figure $1 \mathrm{C})$. Cells positive for NCAM also expressed CD24, a surface antigen known to be a marker of renal progenitor cells in mice $^{22,23}$ and in humans ${ }^{13}$ (Figure 1D). We detected three distinct populations of cells (Figure 1, E and F), the most abundant cells were cells only expressing NCAM. Two minor cell populations consisted of transitional cells co-expressing markers of renal progenitors and podocytes $\left(\mathrm{NCAM}^{+} \mathrm{WT} 1^{+}\right)$, and a subset of more differentiated cells, the parietal podocytes, that no longer ex- pressed NCAM but exhibited the podocyte marker WT1 (Figure 1, E and F). A schematic representation of the cell populations identified in the Bowman's capsule is given in Figure $1 \mathrm{~F}$.

\section{Age-Related Glomerular Changes in Control Wistar Rats}

Changes in renal histology as a function of time was performed in control rats (Figure 2). In renal biopsies of Wistar rats synechiae, focal adhesions of glomerular capillaries to the Bowman's capsule were found between 50 and 60 weeks (Figure 2, B and C). No lesions that were more severe were found in control animals at any other time point (Figure 2, B and C).

\section{Time-Dependent Evolution of Glomerular Lesions in MWF Rats}

Histological analysis of 10-week-old MWF rats revealed early synechiae (Figure 2B) that were not associated with accumulation of type III collagen. From 25 weeks, crescent-like lesions were observed, which increased in number and extension with age (Figure 2B). At an early stage, crescents were characterized by cell proliferation in the absence of type III collagen deposition (Figure 2, D and $H$ ). With time, multilayers of cells accumulated at the site of synechiae, resulting in more severe crescentic lesions, typically found in 40- to 60-week-old MWF (Figure 2, E and F). The percentage of glomeruli with crescents was $39 \% \pm 4 \%$ at 40 weeks, $64 \% \pm 5 \%$ at 50 weeks, and $76 \% \pm 4 \%$ at 60 weeks. Lesions in 40 -week-old rats were characterized by scanty accumulation of type III collagen (Figure 2I). By 50 to 60 weeks, massive, progressive accumulation of type III collagen was observed (Figure $2 \mathrm{~J})$. At the end of the observation period, glomerulosclerosis was so severe as to occupy most of the glomerular tuft in the majority of the glomeruli.

\section{Phenotype of Cell Populations Involved in Crescentic Lesions in MWF Rats}

We then assessed the phenotype of cells contributing to glomerular lesions in MWF rats by evaluating the expression of different markers (Figure 3, A and B). Synechiae in young MWF rats were constituted by claudin $1^{+}$cells (data not shown), which also represented the most abundant cell population in crescents of 60-week-old MWF rats. A high percentage of WT1+ cells was observed in crescents (Figure 3, A and B). The percentage of claudin $1^{+} \mathrm{WT} 1^{+}$cells within crescents was, on average, $12 \%$. Mesangial cells and macrophages, identified respectively by Thy 1.1 and ED1 immunolabeling, represented two minor cell populations in crescents (Figure 3 , $\mathrm{A}$ and $\mathrm{B}$ ). As shown in Figure 4, $\mathrm{NCAM}^{+}$progenitor cells contribute substantially to glomerular extracapillary hyperplastic lesions. 

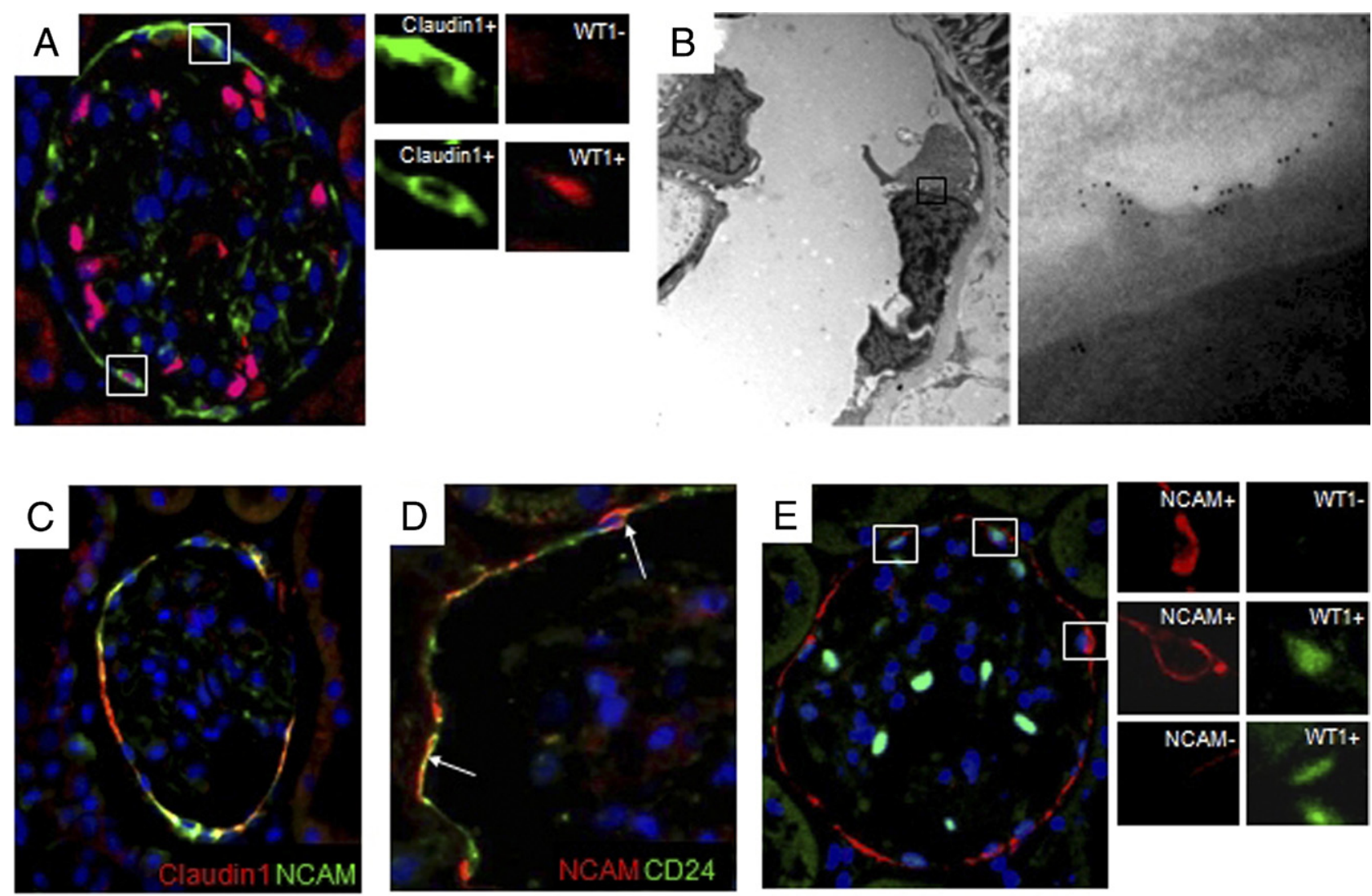

Figure 1. Populations of cells lining the Bowman's capsule of normal adult rat
kidney. A: Representative picture of immunofluorescence staining of claudin1

\section{$\mathrm{F}$}

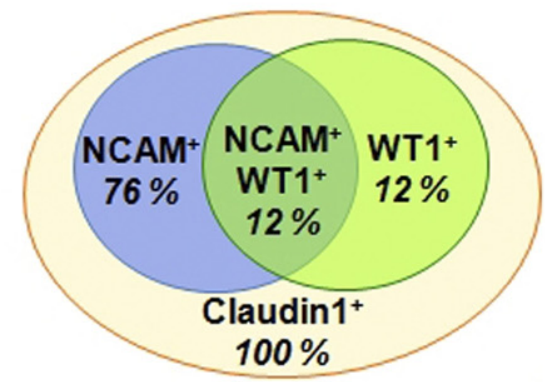

Progenitorcells

Transitional cells kidney. A: Representative picture of immunofluorescence staining of claudin1
(green) and WT1 (red) in the Bowman's capsule of Wistar rats used as controls. (green) and WT1 (red) in the Bowman's capsule of Wistar rats used as controls
High-magnification insets show claudin $1^{+} \mathrm{WT}^{-}$cell (upper insets) and claudin $1^{+} \mathrm{WT}^{+}{ }^{+}$cell (lower insets). B: Representative pattern of immunogold labeling for nephrin in the Bowman's capsule of Wistar rats. Left panel shows the ultrastructure of parietal cells along the Bowman's capsule. High-magnification inset (ltrast ( parietal cells. Original magnification: $\times 4400$ (left panel); $\times 71,000$ (right panel). C: Double immunolabeling for claudin1 (red) and NCAM (green) shows in most of the cells the colocalization of the two markers. D: Double immunofluorescence staining for NCAM (red) and CD24 (green) revealed that in the Bowman's capsule, the two antigens are expressed by the same cells (arrows). E: Representative photomicrograph showing three distinct cell populations in the Bowman's capsule of Wistar rats, ( ${ }^{+} \mathrm{WT}^{-}{ }^{-}$progenito cells (upper inset), $\mathrm{NCAM}^{+} \mathrm{WT}^{+}$transitional cells (middle inset), and $\mathrm{NCAM}^{-} \mathrm{WT}^{+}$parietal podocytes (lower inset). Original magnification, $\times 630$. F Schematic representation of the cell populations identified in the Bowman's capsule of Wistar rats. All of the cells were positive for PEC marker claudin1. A gradient of expression of NCAM and WT1 defined three populations of claudin $1^{+}$PECs with different degrees of differentiation. Seventy-six percent expressed only the progenitor marker NCAM, 12\% expressed NCAM and the podocyte marker WT1, and $12 \%$ both progenitor and podocyte markers.

\section{Treatment with ACEi Reduces Proteinuria and Induces Regression of Crescentic Lesions}

Proteinuria, which positively correlated with the index of crescents $(r=0.94, P<0.01)$, developed with time in MWF rats from 10 weeks and reached values of $730 \pm 44$ mg/day at 60 weeks (Figure 4A). Ten-week treatment with the ACEi lisinopril markedly reduced urinary protein excretion $(159 \pm 26 \mathrm{mg} /$ day, $P<0.01$ versus 60 week old, Figure 4A). When animals were treated only 2 weeks with ACEi (from 50 to 52 weeks of age), proteinuria was only mildly affected, and the index of crescents did not change (50 week old: $1.5 \pm 0.2$ versus treated 52 week old: $1.6 \pm 0.3)$. At 60 weeks, MWF rats had renal function impairment and developed hypertension (serum creati- nine, $2.0 \pm 0.3$ versus controls: $0.7 \pm 0.01 \mathrm{mg} / \mathrm{dL}$, systolic blood pressure: $192.0 \pm 5.2$ versus controls: $87.0 \pm$ $1.7 \mathrm{~mm} \mathrm{Hg}, P<0.01$ ). Treatment with ACEi (from 50 to 60 weeks of age) decreased serum creatinine levels (1.3 \pm $0.1 \mathrm{mg} / \mathrm{dL}, P<0.05)$ and systolic blood pressure $(133.0 \pm 16.8 \mathrm{~mm} \mathrm{Hg}, P<0.05)$ with respect to rats receiving saline. Lisinopril did not limit only the number, but also the extension of the lesions, as demonstrated by the remarkable reduction of the index of crescents, which reflects the degree of severity of the lesions $(P<0.01$, Figure 4A). The extension of glomerular lesions in 60week-old MWF rats receiving lisinopril was even lower than those observed in 50-week-old MWF rats, suggesting the ability of ACEi to induce regression of glomerular injury (Figure 4A). Consistent with the decrease of hyper- 

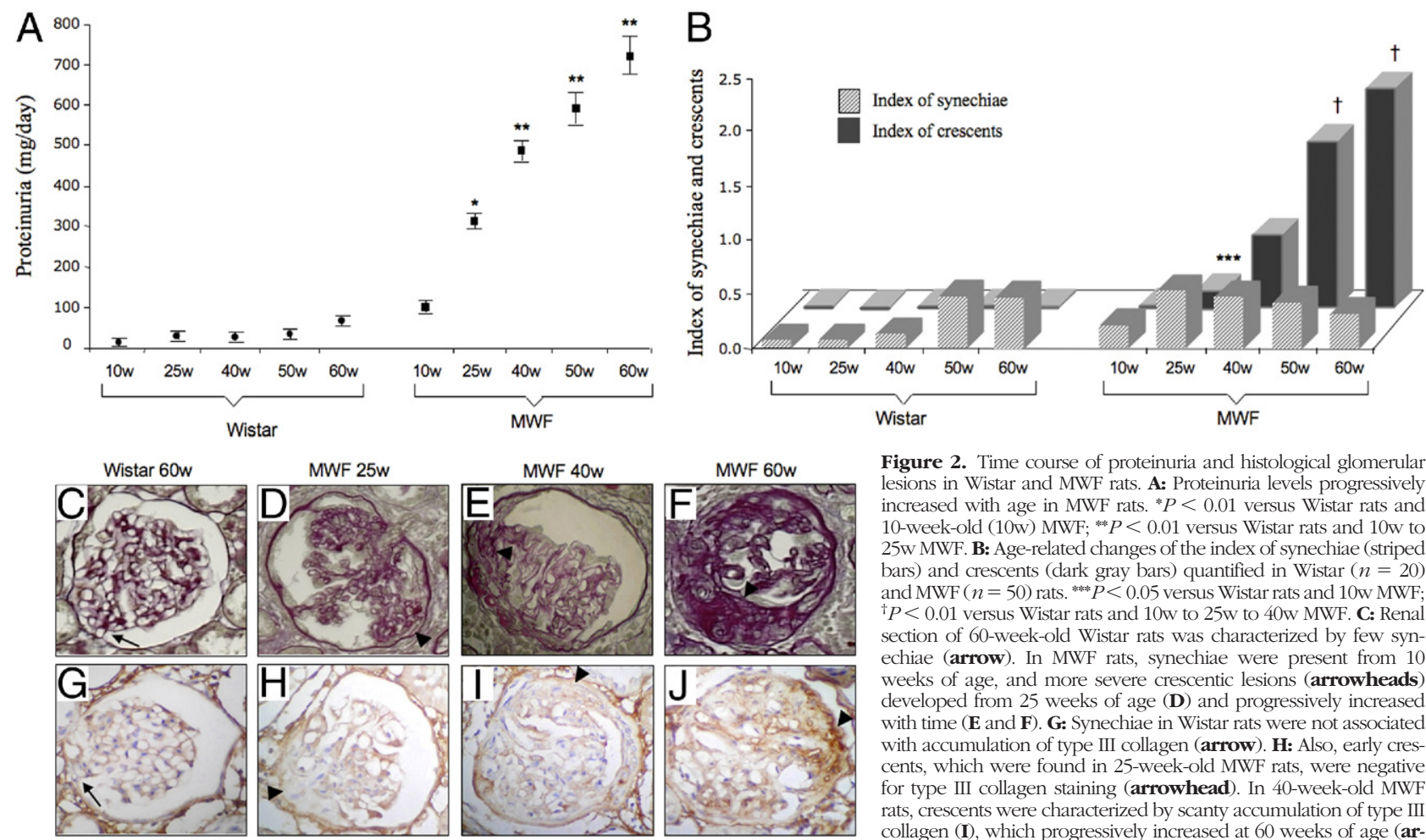

MWF 25W

MWF $40 w$
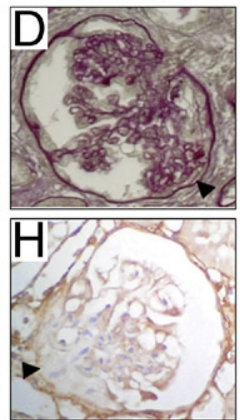

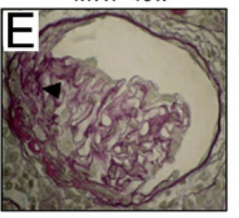

MWF 60w

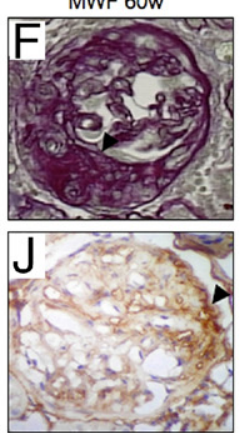

Figure 2. Time course of proteinuria and histological glomerula lesions in Wistar and MWF rats. A: Proteinuria levels progressively increased with age in MWF rats. ${ }^{*} P<0.01$ versus Wistar rats and 10 -week-old (10w) MWF; * * $P<0.01$ versus Wistar rats and $10 \mathrm{w}$ to 25w MWF. B: Age-related changes of the index of synechiae (striped bars) and crescents (dark gray bars) quantified in Wistar $(n=20)$ and MWF $(n=50)$ rats. ${ }^{\text {*wepe }} P<0.05$ versus Wistar rats and $10 \mathrm{~W}$ MWF ${ }^{\dagger} P<0.01$ versus Wistar rats and $10 \mathrm{w}$ to $25 \mathrm{w}$ to $40 \mathrm{w}$ MWF. C: Renal section of 60-week-old Wistar rats was characterized by few synechiae (arrow). In MWF rats, synechiae were present from 10 weeks of age, and more severe crescentic lesions (arrowheads) developed from 25 weeks of age (D) and progressively increased with time ( $\mathbf{E}$ and $\mathbf{F})$. G: Synechiae in Wistar rats were not associated with accumulation of type III collagen (arrow). H: Also, early crescents, which were found in 25-week-old MWF rats, were negative for type III collagen staining (arrowhead). In 40-week-old MWF rats, crescents were characterized by scanty accumulation of type III collagen (I), which progressively increased at 60 weeks of age (arrowheads, $\mathbf{J}$ ). Original magnification, $\times 400$.

plastic lesions, ACEi also reduced type III collagen deposition within crescents (data not shown).

\section{ACE Inhibitor Limits Cell Proliferation in Crescents}

To investigate whether ACEi exerted renoprotection by affecting the proliferative status of cells in crescents, we evaluated the number of BrdU-retaining cells in pulsed MWF animals at 60 weeks of age. Quantification of BrdU ${ }^{+}$ cells revealed that the percentage of proliferating cells within crescents accounted for $9.6 \% \pm 1.5 \%$. The majority of BrdU-labeled cells expressed claudin1 in the absence of podocyte marker WT1, whereas proliferating claudin $1^{+} \mathrm{WT} 1^{+}$and claudin $1^{-} \mathrm{WT} 1^{+}$cells represented rare cell populations in crescents (Figure 4B). ACEi treatment significantly decreased the proliferation of all cell populations, with respect to MWF rats receiving saline (Figure 4B). Consistently, treatment with ACE inhibitor effectively reduced the presence of $\mathrm{NCAM}^{+}$cells in crescents by limiting progenitor cell activation and migration toward capillary tuft, and restored the distribution of progenitor cells along the Bowman's capsule to a pattern similar to controls (Figure 4D).

\section{ACE Inhibitor Preserves Bowman's Capsule Architecture}

The antiproliferative effect of ACEi on cells constituting crescents prompted us to analyze whether this drug

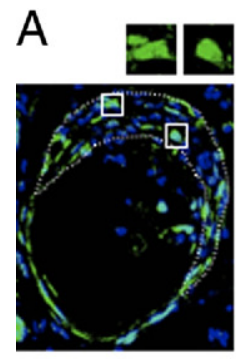

Claudin1

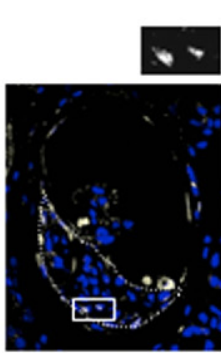

WT1

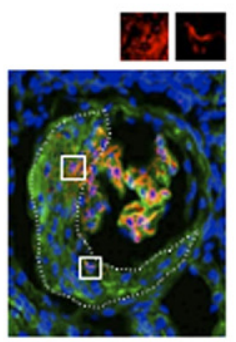

Thy 1.1

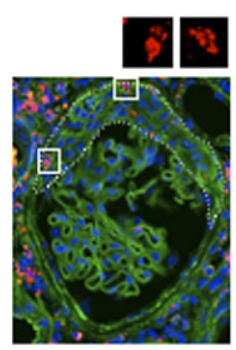

ED1

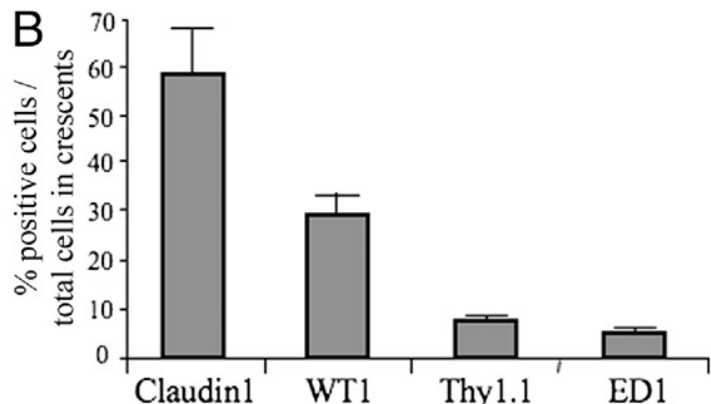

Figure 3. Phenotype of cell populations involved in crescentic lesions. A: Representative pictures of immunofluorescence staining for claudin1 (green), WT1 (white), Thy1.1 (red), and ED1 (red) in glomeruli of 60-week-old MWF rats. High-magnification insets show cells positive for the different markers. Dotted lines define the area of crescents in which positive cells were quantified. Nuclei are stained with DAPI (blue). Original magnification, $\times 630$. B: Quantitative assessment of cells positive for each marker, revealed that the most represented cell population in crescents consisted of claudin $1^{+}$cells, and to a lesser extent of WT1 ${ }^{+}$cells. Thy $1.1^{+}$and $\mathrm{ED} 1^{+}$cells represented minor cell populations within the lesions. 

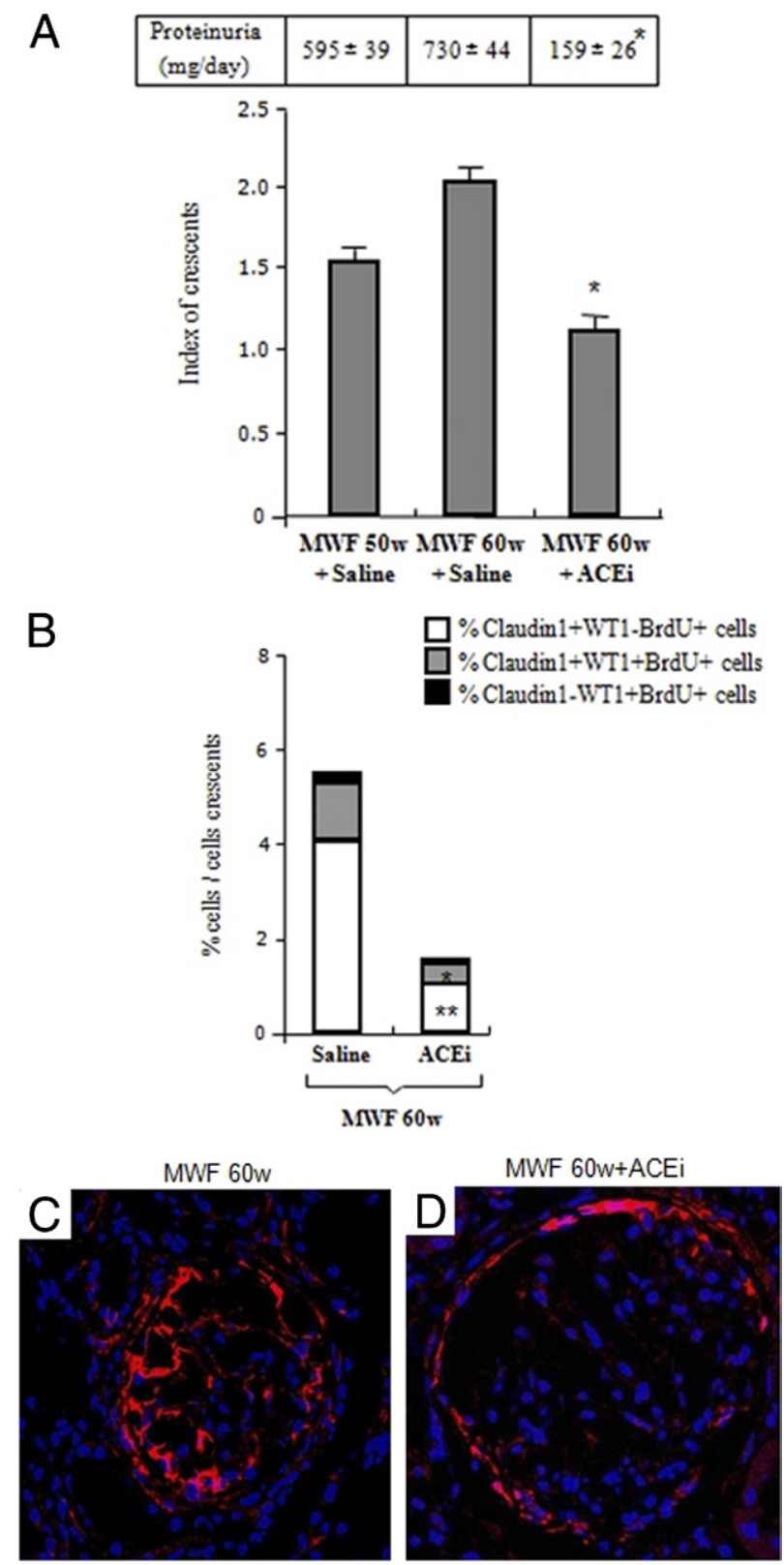

Figure 4. ACE inhibitor reduces the number and extension of crescents and limits progenitor cell activation. A: A significant decrease in proteinuria levels and index of crescents was observed in 60-week-old (60w) MWF rats receiving ACEi for 10 weeks as compared with animals given saline. ${ }^{*} P<0.01$ versus $60 \mathrm{w}$ MWF. B: The percentage of proliferating claudin $1^{+}$WT1 ${ }^{-}$cells (white bars), claudin $1^{+} \mathrm{WT}^{+}{ }^{+}$cells (gray bars), and claudin $1^{-} \mathrm{WT}^{+}{ }^{+}$cells (black bars) was lower in crescents of ACEi-treated MWF rats with respect to animals given saline. Cell proliferation was assessed by quantification of the number of $\mathrm{BrdU}^{+}$cells in pulse-chased MWF rats. ${ }^{* * *} P<0.05$ versus $60 \mathrm{w}$ MWF; ${ }^{*} P<0.01$ versus $60 \mathrm{w}$ MWF. C: The expression of NCAM, which identifies renal progenitor cells, was evaluated in crescentic lesions of 60 -week-old MWF rats. Note that $\mathrm{NCAM}^{+}$cells (red) were localized in Bowman's capsule and capillary tuft, as well as in crescents. D: ACEi effectively reduced the presence of progenitor cells in crescents and restored the distribution of $\mathrm{NCAM}^{+}$cells in the Bowman's capsule to the pattern observed in controls (shown in Figure 1). Nuclei are stained with DAPI (blue). Original magnification, $\times 630$.

could also influence the proliferative status of cells in the Bowman's capsule. The total number of cells lining the Bowman's capsule was comparable in 60-week-old MWF rats $\left(445.8 \pm 19.8 \times 10^{-4} /\right.$ length of capsule) and controls (427.2 $\pm 5.2 \times 10^{-4} /$ length of capsule), as previously re- ported. ${ }^{10}$ In 60-week-old MWF rats, the percentage of claudin $1^{+}$WT1 ${ }^{-}$PECs was markedly $(P<0.05)$ enhanced and claudin $1^{+} \mathrm{WT} 1^{+}$cells significantly decreased $(P<$ 0.05 , Figure $5 \mathrm{~A}$ ) with respect to controls. The proliferation of claudin $1^{+}$PECs $\left(\mathrm{BrdU}^{+}\right)$in the Bowman's capsule of MWF rats was markedly $(P<0.05)$ enhanced as compared with controls (Figure 5, B and C), in which proliferating claudin $1^{+}$

\section{A $\square \%$ Claudin $1+$ WT1- cells B}
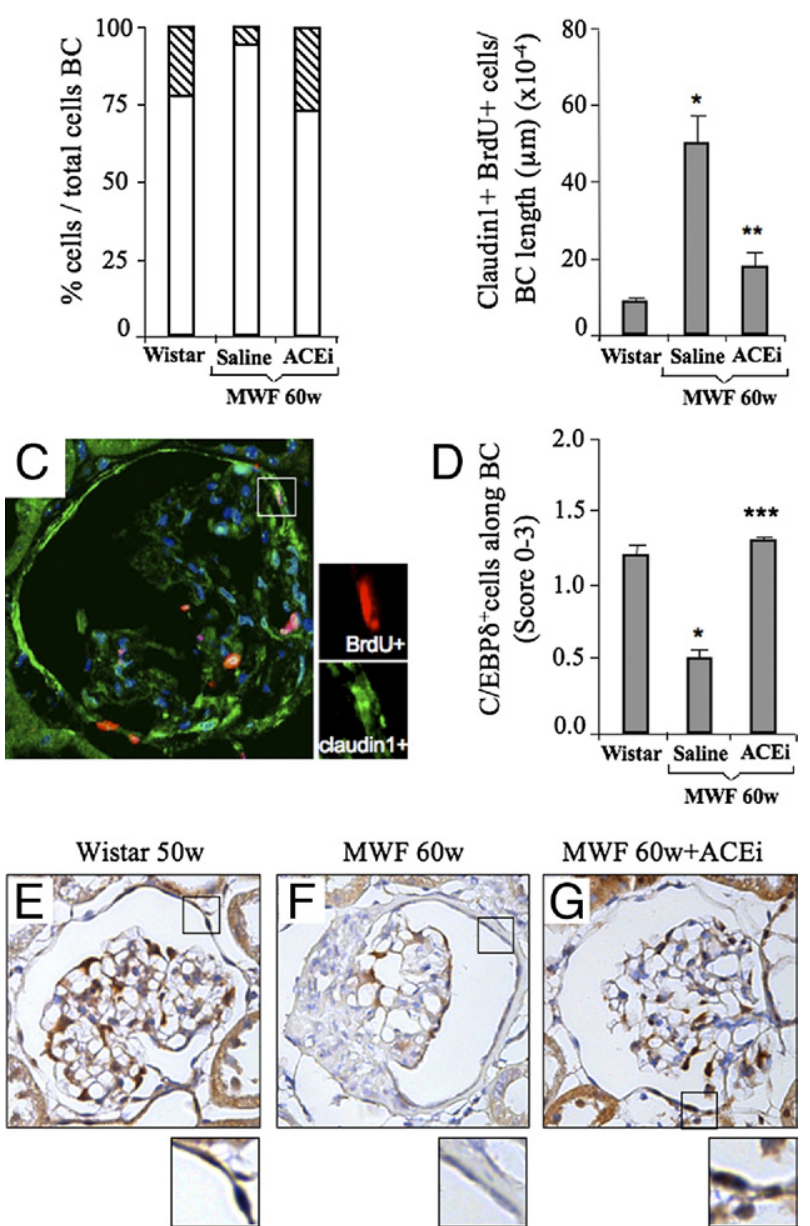

Figure 5. ACE inhibitor reduces proliferation of Bowman's capsule cells and regulates the expression of the cell cycle inhibitor $\mathrm{C} / \mathrm{EBP} \delta$. A: In the Bowman's capsule of 60-week-old MWF rats given saline, the percentage of claudin $1^{+} \mathrm{WT}^{-}$cells (white bars) was markedly enhanced, and claudin $1^{+}$WT1 ${ }^{+}$cells (striped bars) decreased with respect to Wistar rats. ACEi restored the percentage of both cell populations to control levels. B: Cell proliferation of claudin $1^{+}$cells along the Bowman's capsule was enhanced in 60-week-old MWF rats given saline as compared with controls. ACEi treatment significantly limited the number of proliferating cells within the Bowman's capsule (claudin $1^{+} \mathrm{BrdU}^{+}$). ${ }^{*} P<0.05$ versus Wistar; ${ }^{* *} P<$ 0.01 versus $60 \mathrm{w}$ MWF + saline. C: Representative picture of immunofluorescence staining for claudin1 (green) and BrdU (red) in the Bowman's capsule of 60-week-old MWF rats. Inset shows a cell expressing both markers. Nuclei are stained with DAPI (blue). Original magnification, $\times 630$. D: The expression of $\mathrm{C} / \mathrm{EBP} \delta$ in the Bowman's capsule, evaluated by semiquantitative score, decreased at 60 weeks of age in MWF rats receiving saline as compared with Wistar rats, and was normalized by ACEi treatment. ${ }^{*} P<0.05$ versus Wistar; ${ }^{* 0 * a \cdot} P<0.05$ versus $60 \mathrm{w}$ MWF + saline. Representative photomicrographs of C/EBP $\delta$ expression in Wistar $(\mathbf{E})$ and MWF rats given saline $(\mathbf{F})$ or treated with $\mathrm{ACEi}(\mathbf{G})$ are shown. Note that in ACEi-treated rats, as in Wistar rats, most of the cells were positive for $\mathrm{C} / \mathrm{EBP} \delta$ (brown signal at high magnification); in MWF rats, the majority of the cells did not express the marker. Nuclei (blue) were counterstained with hematoxylin. Original magnification, $\times 400$. 

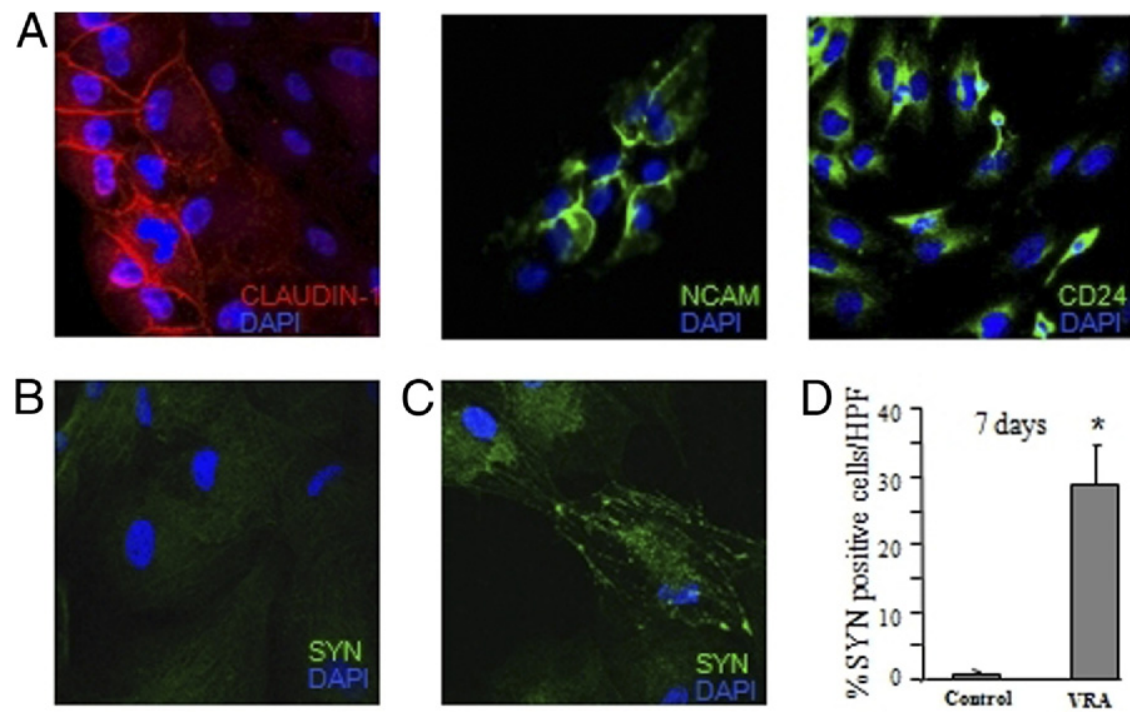

Control

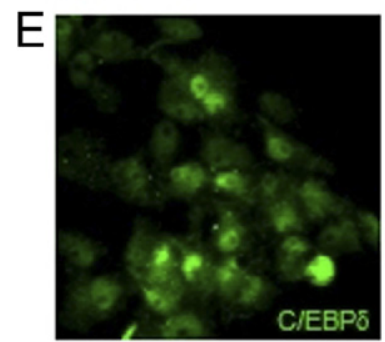

Ang II (6h)

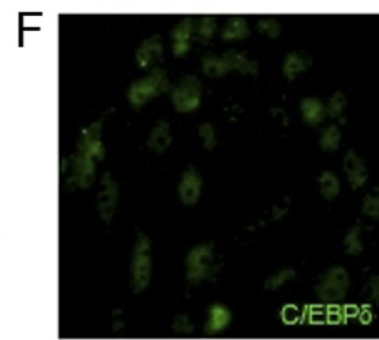

Control

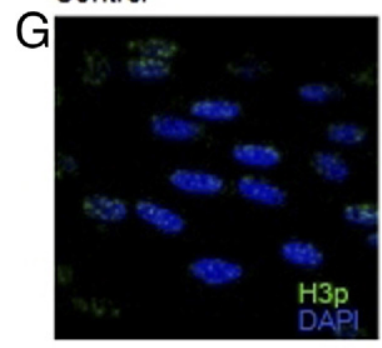

Ang II (24h)

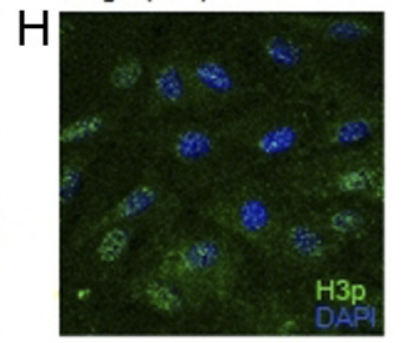

\section{1}

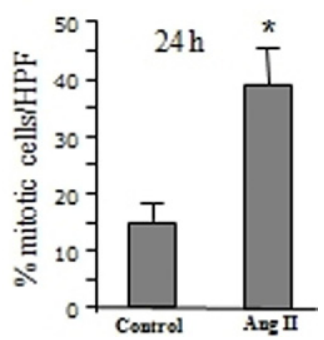

Figure 6. Characterization, differentiation, and proliferation of cultured PECs. A: Representative images of PECs, obtained from initial outgrowth of capsulated control rat glomeruli, that constitutively expressed claudin1 (red), NCAM (green), or CD24 (green). Cells were co-stained with DAPI (blue). B and C: Immunofluorescence micrographs of immunoisolated claudin $1^{+}$PECs co-stained with synaptopodin (SYN, green) and DAPI (blue). No signal for SYN (B) was observed in control cells. Cells treated with VRAD medium for 7 days expressed the podocyte marker SYN (C). D: Quantification of $\mathrm{SYN}^{+}$ cells: data are expressed as percentage of fluorescent cells per total $\mathrm{DAPI}^{+}$cells in each $\mathrm{HPF}$ ${ }^{*} P<0.001$ versus control. E and F: PECs show a constitutive nuclear expression of the transcription factor $\mathrm{C} / \mathrm{EBP} \delta$ (green; $\mathbf{E}$ ), that was markedly reduced after 6-hour incubation with angiotensin II (Ang II) (F). G and H: Representative images of H3p (green) and DAPI (blue) in immunoisolated claudin $1^{+}$PECs untreated $(\mathbf{G})$ or treated (H) with Ang II for 24 hours. Nuclear $\mathrm{H} 3 \mathrm{p}$ expression was markedly increased in cells exposed to Ang II (H) with respect to control cells (G). I: Quantification of mitotic cells: data are expressed as percentage of mitotic cells per total DAPI-positive cells in each HPF. ${ }^{*} P<0.001$ versus control. All images were obtained at original magnification, $\times 630$.
PECs were extremely rare. Consistent with the ability of ACEi to reduce cell proliferation in crescents, the treatment also reduced the proliferative status of Bowman's capsule cells (Figure 5B). The effect of ACEi to limit cell proliferation translated into normalization of the percentages of clau$\operatorname{din} 1^{+}$PECs and parietal podocytes (claudin $1^{+} \mathrm{WT} 1^{+}$) in the Bowman's capsule (Figure 5A).

\section{Mechanism Underlying the Renoprotective Effect of ACEi: Role of the Transcription Factor $C / E B P \delta$}

To investigate the mechanism by which ACEi limited the abnormal proliferation of Bowman's capsule cells, we evaluated the role of $\mathrm{C} / \mathrm{EBP} \delta$. C/EBP $\delta$ is a transcription factor specific for adult cells and mitotically quiescent stem cells of epithelial origin, ${ }^{24}$ which regulates cell cycle and selfrenewal potential. ${ }^{25,26}$ In Wistar rats, C/EBP $\delta$ was detect- able in the majority of cell nuclei along the Bowman's capsule (Figure 5, D and E), indicating the low-cycling status of these cells, whereas it was markedly decreased in MWF rats at 60 weeks $(P<0.05$, Figure 5, D and F). ACEi restored $\mathrm{C} / \mathrm{EBP} \delta$ expression to control levels $(P<0.05$, Figure 5, D and $G$ ), suggesting the involvement of C/EBP $\delta$ in the antiproliferative effect of this drug.

\section{PEC Characterization and Differentiation}

Parietal epithelial cells were obtained by the initial outgrowth of capsulated control rat glomeruli, as previously described. ${ }^{12,23}$ After 5-day culture, cells outgrown from adherent glomeruli were harvested and cultured. Cultured PECs expressed either markers of adult epithelial cells or immature progenitors including claudin1, NCAM, and CD24 (Figure 6A), as it occurred in vivo in renal tissue (Figure 1). 
The capacity of PECs to differentiate and generate podocytes was further investigated by exposing cells to a specific inductive medium, as previously described. ${ }^{13}$ Specifically, immunoisolated claudin $1^{+}$PECs incubated for 7 days with VRAD medium became positive for synaptopodin with the typical localization along cytoskeletal fibers (Figure 6, B and C). At this time, 29\% of cells acquired the specific podocyte marker (Figure 6D), thus supporting the concept that PECs contain a population of progenitor cells devoted to generate podocytes.

To evaluate whether angiotensin II could affect the proliferative activity of parietal epithelial cells, the involvement of the transcription factor $\mathrm{C} / \mathrm{EBP} \delta$ in the regulation of PECs proliferation was studied. Incubation with angiotensin II markedly reduced the expression of $\mathrm{C} / \mathrm{EBP} \delta$ (Figure 6, E and F), suggesting that the mitogenic effect of the peptide could be exerted through the down-regulation of the C/EBP $\delta$ signaling pathway. As a marker of mitosis, we assessed the phosphorylated form of Histone H3-serine $10(\mathrm{H} 3 \mathrm{p})$ on immunoisolated claudin $1^{+}$PECs incubated with angiotensin II for 24 hours. As shown in Figure $6 \mathrm{H}$ a large number of PECs revealed nuclearpositive granular staining for $\mathrm{H} 3 \mathrm{p}$ on angiotensin II exposure with respect to unstimulated cells (Figure 6G). The percentage of proliferating cells induced by angiotensin II averaged $39 \% \pm 6 \%$ as compared to $15 \% \pm 3 \%$ observed in unstimulated cells (Figure 6I).

\section{Discussion}

Here, we describe for the first time a population of renal progenitor cells within the Bowman's capsule of adult rat kidney. Previous studies in rodents showed that parietal epithelial cells migrate into the glomerular tuft and differentiate into podocytes. ${ }^{11} \mathrm{~A}$ heterogeneous population of cells expressing variable levels of stemness markers, CD24 and CD133, was also found in the Bowman's capsule of normal human kidney. ${ }^{12}$ In rats, however, no stem cell population in the Bowman's capsule has been described so far. CD133 proteins do not identify progenitor cells due to interspecies differences, which prompted us to search for another stemness marker. One of us had previously discovered neural cell adhesion molecule (NCAM), normally expressed in rodent and human metanephric mesenchyme, ${ }^{27,21}$ in the Bowman's capsule of the mature kidney. ${ }^{20}$ At that time, the possibility that stem cells existed along the Bowman's capsule was not conceived. Here, we show that NCAM is expressed by the large majority (about $88 \%$ ) of PECs (claudin $1^{+}$cells), and that $\mathrm{NCAM}^{+}$cells coexpress CD24, a marker of stemness in human and mouse kidney. In agreement with findings in humans, ${ }^{13}$ co-staining with NCAM and the podocyte marker WT1 in the Bowman's capsule of normal rats allowed to identify three distinct cell populations of epithelial origin. They include immature progenitor cells expressing only NCAM, transitional cells expressing markers for both progenitor cells and podocytes $\left(\mathrm{NCAM}^{+} \mathrm{WT}^{+}{ }^{+}\right)$, and more differentiated epithelial cells, the parietal podocytes $\left(\mathrm{NCAM}^{-} \mathrm{WT} 1^{+}\right)$. The fact that parietal podocytes retained the expression of claudin 1 but lost NCAM supports the notion that this latter marker exclusively characterizes immature cells in rats. On the other hand, finding that cultured PECs expressing claudin1 and NCAM, when exposed to an appropriate inductive medium, can acquire phenotypic features of differentiated podocytes, further supports the notion that $\mathrm{NCAM}^{+}$cells represent a progenitor cell population.

Munich Wistar Frömter rats were instrumental here to document the contribution of progenitors and differentiated cells during the evolution of glomerular lesions. Early abnormalities were bridges between parietal and visceral epithelium, followed by extracapillary crescentic lesions that eventually evolved to fibrosis.

The majority of cells in synechiae and crescents was represented by parietal epithelial cells of the Bowman's capsule (claudin ${ }^{+}$PECs). Podocytes were present at a low extent only in hyperplastic lesions. In rats pulsechased with BrdU, both claudin $1^{+}$PECs and podocytes $\left(\mathrm{WT}^{+}\right)$within crescents proliferated although the percentage of mitotically active cells was low, reflecting the late stage of the disease. There is controversy on the cellular composition of crescents in glomerular disease. Lineage tracing experiments of either parietal epithelial cells or podocytes indicated the former as the predominant population responsible for proliferative lesions, with a lower contribution of podocytes. ${ }^{28}$ By contrast, others documented that podocytes represent an integral cellular component of crescents ${ }^{14,29-31}$ that transform from a terminally differentiated nondividing to a migratory cycling cell. ${ }^{29}$ Here, the presence in crescents of a high percentage of claudin $1^{+}$cells indicates that cells of parietal origin acquired a migratory and proliferative activity during the progression of the disease.

In the Bowman's capsule, parietal epithelial cells represent a reservoir of cells that contribute to podocyte physiological turnover, ${ }^{13}$ as reflected by the normal endowment with claudin $1^{+}$PECs and parietal podocytes in control rats. In old MWF animals, claudin $1^{+}$PECs increased in number at the expense of parietal podocytes, and they actively proliferated, reflecting dysregulation of their ability to generate podocytes and to repair injury. It is also possible that bridges of dysfunctional podocytes with the Bowman's basement membrane, activated parietal epithelial cells, including $\mathrm{NCAM}^{+}$progenitor cells, to migrate. ${ }^{32}$ Finding of a high number of $\mathrm{NCAM}^{+}$cells in crescents indicates the migration of renal progenitors toward the glomerular tuft. This would explain previous evidence of renal progenitor cells in hyperplastic lesions of patients with podocytopathies associated with crescents and glomerulosclerosis. ${ }^{14}$

Another unprecedented result of the present paper is that ACEi, beside the well-known effect of lowering blood pressure and proteinuria, limited the formation of crescents, preventing the accumulation of extracellular matrix and the evolution toward glomerulosclerosis. The reparative process induced by ACEi was accomplished through a marked reduction of parietal epithelial cell and podocyte proliferation both in crescents and in the Bowman's capsule. Re-establishment of normal glomerular architecture by ACE inhibition was associated with reduced activation of $\mathrm{NCAM}^{+}$progenitors and restoration 
of their distribution along the Bowman's capsule. These data concur to indicate parietal epithelial cells as a novel cellular target of ACEi therapy.

The present study also provides a mechanism through which the ACEi fosters glomerular regeneration. The presence of most cells along the Bowman's capsule expressing the cell cycle inhibitor $\mathrm{C} / \mathrm{EBP} \delta^{24}$ in control rats indicated the low proliferative status of parietal epithelial cells, consistent with the low number of BrdU-labeled cells in the glomerular capsule. In MWF rats, the scanty expression of C/EBP $\delta$ in the Bowman's capsule accounted for the high proliferative rate of claudin $1^{+}$PECs. The role of C/EBP $\delta$ in maintaining the progenitors in a quiescent state was confirmed by its marked constitutive expression in cultured $\mathrm{NCAM}^{+}$PECs isolated from capsulated glomeruli. That angiotensin II decreased C/EBP $\delta$ expression and markedly stimulated mitosis of cultured PECs highlights the ability of the peptide to enhance PEC activation status leading to uncontrolled proliferation. The restoration of C/EBP $\delta$ expression in the Bowman's capsule by ACEi indicated a path through which the drug directly limits the activation of parietal progenitor cells, offering a candidate molecular target for pharmacological induction of glomerular healing.

In summary, we have: i) described in the rat kidney the existence of three types of glomerular epithelial cells at different stages of differentiation from immature progenitor cells to differentiated parietal podocytes; ii) demonstrated that cultured PECs with immature phenotype can generate podocytes; iii) identified the phenotype of proliferating cells within lesions; iv) documented in a single model a sequence of pathological events linking synechiae to crescents and glomerulosclerosis; and v) provided evidence that ACEi induces renal repair by modulating renal progenitor cell proliferation and migration and restoring the glomerular architecture.

These data provide a clue for designing specific molecules targeted to novel players of renal repair that can possibly foster the intrinsic capacity of the kidney to regenerate.

\section{Acknowledgments}

We thank Dr. Fabio Sangalli for excellent technical assistance and Dr. Antonella Piccinelli for work on figures. We are indebted to Prof. Paola Romagnani, Dr. Daniela Macconi, and Prof. Malcolm Alison for helpful extensive discussion. The 5B8 monoclonal antibody was obtained from Developmental Studies Hybridoma Bank, developed under the auspices of NICHD, and maintained by the Department of Biological Sciences, University of lowa, lowa City. Manuela Passera helped prepare the manuscript.

\section{References}

1. Just PM, Riella MC, Tschosik EA, Noe LL, Bhattacharyya SK, de Charro F: Economic evaluations of dialysis treatment modalities. Health Policy 2008, 86:163-180

2. Gridelli B, Remuzzi G: Strategies for making more organs available for transplantation. N Engl J Med 2000, 343:404-410
3. Denton KM, Anderson WP, Sinniah R: Effects of angiotensin II on regional afferent and efferent arteriole dimensions and the glomerular pole. Am J Physiol Regul Integr Comp Physiol 2000, 279:R629-R638

4. Anderson S, Meyer TW, Rennke HG, Brenner BM: Control of glomerular hypertension limits glomerular injury in rats with reduced renal mass. J Clin Invest 1985, 76:612-619

5. Remuzzi A, Fassi A, Bertani T, Perico N, Remuzzi G: ACE inhibition induces regression of proteinuria and halts progression of renal damage in a genetic model of progressive nephropathy. Am J Kidney Dis 1999, 34:626-632

6. Remuzzi G, Benigni A, Remuzzi A: Mechanisms of progression and regression of renal lesions of chronic nephropathies and diabetes. J Clin Invest 2006, 116:288-296

7. Remuzzi A, Gagliardini E, Sangalli F, Bonomelli M, Piccinelli M, Benigni A, Remuzzi G: ACE inhibition reduces glomerulosclerosis and regenerates glomerular tissue in a model of progressive renal disease. Kidney Int 2006, 69:1124-1130

8. Joles JA, Braam B, Verhaar MC: ACE inhibition and glomerular repair: restructuring or regeneration? Kidney Int 2006, 69:1105-1107

9. Oliver JA: Adult renal stem cells and renal repair. Curr Opin Nephrol Hypertens 2004, 13:17-22

10. Macconi D, Sangalli F, Bonomelli M, Conti S, Condorelli L, Gagliardin E, Remuzzi G, Remuzzi A: Podocyte repopulation contributes to regression of glomerular injury induced by ACE inhibition. Am J Pathol 2009, 174:797-807

11. Appel D, Kershaw DB, Smeets B, Yuan G, Fuss A, Frye B, Elger M, Kriz W, Floege J, Moeller MJ: Recruitment of podocytes from glomerular parietal epithelial cells. J Am Soc Nephrol 2009, 20:333-343

12. Sagrinati C, Netti GS, Mazzinghi B, Lazzeri E, Liotta F, Frosali F, Ronconi E, Meini C, Gacci M, Squecco R, Carini M, Gesualdo L, Francini F, Maggi E, Annunziato F, Lasagni L, Serio M, Romagnani S, Romagnani P: Isolation and characterization of multipotent progenitor cells from the Bowman's capsule of adult human kidneys. J Am Soc Nephrol 2006, 17:2443-2456

13. Ronconi E, Sagrinati C, Angelotti ML, Lazzeri E, Mazzinghi B, Ballerini L, Parente E, Becherucci F, Gacci M, Carini M, Maggi E, Serio M, Vannelli GB, Lasagni L, Romagnani S, Romagnani P: Regeneration of glomerular podocytes by human renal progenitors. J Am Soc Nephrol 2009, 20:322-332

14. Smeets B, Angelotti ML, Rizzo P, Dijkman H, Lazzeri E, Mooren F, Ballerini L, Parente E, Sagrinati C, Mazzinghi B, Ronconi E, Becherucci F, Benigni A, Steenbergen E, Lasagni L, Remuzzi G, Wetzels J, Romagnani P: Renal progenitor cells contribute to hyperplastic lesions of podocytopathies and crescentic glomerulonephritis. J Am Soc Nephrol 2009, 20:2593-2603

15. Remuzzi A, Puntorieri S, Battaglia C, Bertani T, Remuzzi G: Angiotensin converting enzyme inhibition ameliorates glomerular filtration of macromolecules and water and lessens glomerular injury in the rat. J Clin Invest 1990, 85:541-549

16. Vasiliades J: Reaction of alkaline sodium picrate with creatinine: $\mathrm{i}$. Kinetics and mechanism of formation of the mono-creatinine picric acid complex. Clin Chem 1976, 22:1664-1671

17. Takano Y, Yamauchi K, Hiramatsu N, Kasai A, Hayakawa K, Yokouch M, Yao J, Kitamura M: Recovery and maintenance of nephrin expression in cultured podocytes and identification of HGF as a repressor of nephrin. Am J Physiol Renal Physiol 2007, 292:F1573-1582

18. Lasagni L, Ballerini L, Angelotti ML, Parente E, Sagrinati C, Mazzinghi B, Peired A, Ronconi E, Becherucci F, Bani D, Gacci M, Carini M, Lazzeri E, Romagnani P: Notch activation differentially regulates renal progenitors proliferation and differentiation toward the podocyte lineage in glomerular disorders. Stem Cells 2010, 28:1674-1685

19. Ohse T, Pippin JW, Vaughan MR, Brinkkoetter PT, Krofft RD, Shankland SJ: Establishment of conditionally immortalized mouse glomerular parietal epithelial cells in culture. J Am Soc Nephrol 2008, 19 : 1879-1890

20. Abbate M, Brown D, Bonventre JV: Expression of NCAM recapitulates tubulogenic development in kidneys recovering from acute ischemia. Am J Physiol 1999, 277:F454-463

21. Bard JB, Gordon A, Sharp L, Sellers WI: Early nephron formation in the developing mouse kidney. J Anat 2001, 199:385-392

22. Challen GA, Martinez G, Davis MJ, Taylor DF, Crowe M, Teasdale RD, Grimmond SM, Little MH: Identifying the molecular phenotype of renal progenitor cells. J Am Soc Nephrol 2004, 15:2344-2357 
23. Swetha G, Chandra V, Phadnis S, Bhonde R: Glomerular parietal epithelial cells of adult murine kidney undergo EMT to generate cells with traits of renal progenitors. J Cell Mol Med 2009, (In press)

24. Barbaro V, Testa A, Di Iorio E, Mavilio F, Pellegrini G, De Luca M: $\mathrm{C} / \mathrm{EBP} d e l t a$ regulates cell cycle and self-renewal of human limbal stem cells. J Cell Biol 2007, 177:1037-1049

25. O'Rourke J, Yuan R, DeWille J: CCAAT/enhancer-binding proteindelta (C/EBP-delta) is induced in growth-arrested mouse mammary epithelial cells. J Biol Chem 1997, 272:6291-6296

26. Zhang Y, Liu T, Yan P, Huang T, Dewille J: Identification and characterization of CCAAT/Enhancer Binding proteindelta (C/EBPdelta) target genes in G0 growth arrested mammary epithelial cells. BMC Mol Biol 2008, 9:83

27. Metsuyanim S, Harari-Steinberg O, Buzhor E, Omer D, PodeShakked N, Ben-Hur H, Halperin R, Schneider D, Dekel B: Expression of stem cell markers in the human fetal kidney. PLoS One 2009, 4:e6709
28. Smeets B, Uhlig S, Fuss A, Mooren F, Wetzels JF, Floege J, Moeller $\mathrm{MJ}$ : Tracing the origin of glomerular extracapillary lesions from parietal epithelial cells. J Am Soc Nephrol 2009, 20:2604-2615

29. Thorner PS, Ho M, Eremina V, Sado Y, Quaggin S: Podocytes contribute to the formation of glomerular crescents. J Am Soc Nephrol 2008, 19:495-502

30. Bariety J, Nochy D, Mandet C, Jacquot C, Glotz D, Meyrier A: Podocytes undergo phenotypic changes and express macrophagic-associated markers in idiopathic collapsing glomerulopathy. Kidney Int 1998, 53:918-925

31. Moeller MJ, Soofi A, Hartmann I, Le Hir M, Wiggins R, Kriz W, Holzman LB: Podocytes populate cellular crescents in a murine model of inflammatory glomerulonephritis. J Am Soc Nephrol 2004, 15:61-67

32. Le Hir M, Keller C, Eschmann V, Hahnel B, Hosser H, Kriz W: Podocyte bridges between the tuft and Bowman's capsule: an early event in experimental crescentic glomerulonephritis. J Am Soc Nephrol 2001, 12:2060-2071 